\title{
Adaptive soft computing methods for control of hemodialysis machines
}

\author{
József Klespitz*, Márta Takács*, Imre Rudas*, Levente Kovács* \\ * Óbuda University, Budapest, Hungary \\ klespitz.jozsef@phd.nui-obuda.hu, \{takacs.marta,kovacs.levente\}@nik.uni-obuda.hu, rudas@uni-obuda.hu
}

\begin{abstract}
In hemodialysis systems the control of peristaltic pumps can be vital as these machines support and maintain life functions. In this paper different soft computing approaches are compared for the strict control of peristaltic pumps. An adaptive fuzzy logic and an adaptive neuro-fuzzy inference system are examined then compared with a classical PID controller. A fast controller is sought, which is insensitive in speak of disturbances and has no residual error. Finally, experiences of the designed controller on a real system are detailed in the paper.
\end{abstract}

\section{INTRODUCTION}

If the kidney of a person cannot perform its purpose or does not function at all, then external help is necessary by hemodialysis machines. These are filtering the blood of the patient and removing the unnecessary components [1]. In hemodialysis machines peristaltic pumps are responsible for fluid transport, such as blood transfer and removal of ultrafiltration $[2,3,4]$. In peristaltic pumps the transferred fluid gets in contact only with the inside of the tubing, where the tubing can be replaced if necessary. Thus, disposable kits can be created, which keeps the sterility of the transported fluid. Furthermore, peristaltic pumps are gentle to the transported liquid, and in case of blood pumps the chances of hemolysis are reduced due to the lower shearing forces [5].

Peristaltic pumps typically comprises from housing (also called manifold), from an elastic tube segment (continued with the tubing) and a pump head, built-up from a central rotor and at least two rollers. During operation one roller closes the tube segment by pressing the tube segment to the housing. By rotation the roller creates a pressure wave and the fluid flows in the tubing. Before the first roller reaches the end of the housing and release the manifold, the second roller will close tube segment preventing by this way back-flow [6].

The accuracy of these pumps mainly depends on the accuracy of the volume of the tube segment. As the deviation of production of the tube segment is relevant, the difference in transfer volume can reach $\pm 10 \%$ according to the expected one [7]. This error is particularly relevant at higher flow rates. As peristaltic pumps transfer blood, medical fluids, sometime drugs and they are responsible for the patient fluid balance hence, a strict control is necessary $[5,8]$.

In previous works a hemodialysis machine model was identified and control possibilities were compared both with classical and basic soft computing methods [9, 10]. Practical implementation was tested as well.
The goal of this paper is to involve advanced softcomputing control methods in the research, on an improved hemodialysis model. The main goal is to find a lightweight, fast controller, which is adaptive (in speak of the tube segment) and has no residual error. Therefore adaptive neuro-fuzzy inference systems (ANFIS) are designed and compared to the previous results $[9,10]$. The ANFIS controller is tested on a real hemodialysis system as well.

\section{METHOD}

\section{A. Former results}

In one of our previous works [9], the hemodialysis system was identified:

$$
\mathrm{H}(\mathrm{s})=\mathrm{K}_{\text {pump }} / \mathrm{s}
$$

The skeleton of the model used for simulations is the same as in [9] (Fig. 1). The input of the system is the desired flow. At first, this is attached to the first plant. Here the plant contains the identified transfer function and on its output the ideal transfer volume appears. Meanwhile, the ideal flow is attached to the second plant. At this point slope error can appear in the system, which refers to the deviation of production of the tube segment. With the use of this modified flow the real transfer volume arise; furthermore, this volume can be burdened with further errors. Addition of constant offset error can simulate the transfer error, while addition of a sine wave can simulate uncertainty in weight measurement. As a result the real transfer volume can be coupled out from the second plant.

The difference of the real and ideal transfer volumes will result in the error signal. The error signal is connected to the controller, which calculates the control signal. Finally, the control signal intervenes to the real system and eliminates the introduced errors. The advantage of this model is that the controller can be easily replaced; hence, it provides a fast and simple way for testing.

However, this model was partially redesigned. The saturation applied on the modified flow was changed and also the glue logic between the output of the controller and the place of intervention had some conceptual modification [10]. As a result a more accurate model was created, relevantly changing its basic behavior. 


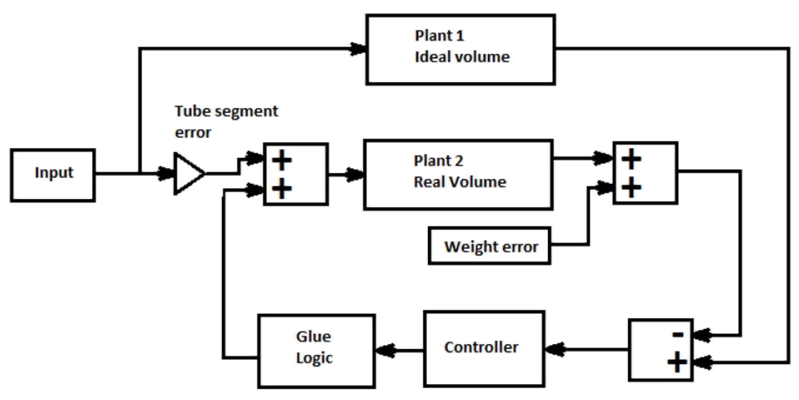

Figure 1. Schematic of examined model

The controllers were previously tested by

- settling time,

- overshoot,

- accuracy

- robustness.

\section{B. Designed controllers}

In [8] the PID controller was designed for a $61^{\circ}$ phase margin. Moreover, we have previously tested the relevance of basic fuzzy systems, but most of them were problematic as residual error could not be eliminated. However, by having an adaptive fuzzy system the fuzzy decision making system could get the integral of the error signal as input completed with an anti-windup logic. Furthermore on the output of the fuzzy logic an iterative learning control method can be applied. The performance of such a system was comparable with classical, PID-type controllers and also removed the residual error [10].

As a result, we have oriented on adaptive neuro-fuzzy inference systems (ANFIS).

ANFIS systems use a layered structure (Fig. 2.). The task is to define the suitable membership functions for fuzzyfication and defuzzyfication (for the "if" and "then" parts) and to teach trough training the rules for the decision making subsystem (in this case the parameters for the neurons) $[15,16]$.



Figure 3. The structure of mANFIS

The second designed controller follows the structure of the adaptive fuzzy system from [10]. However, in this case the fuzzy logic is replaced with an ANFIS (modified ANFIS - mANFIS). The input (error signal) is integrated through an anti-windup system, and this is connected to the ANFIS. On the output of the ANFIS iterative learning

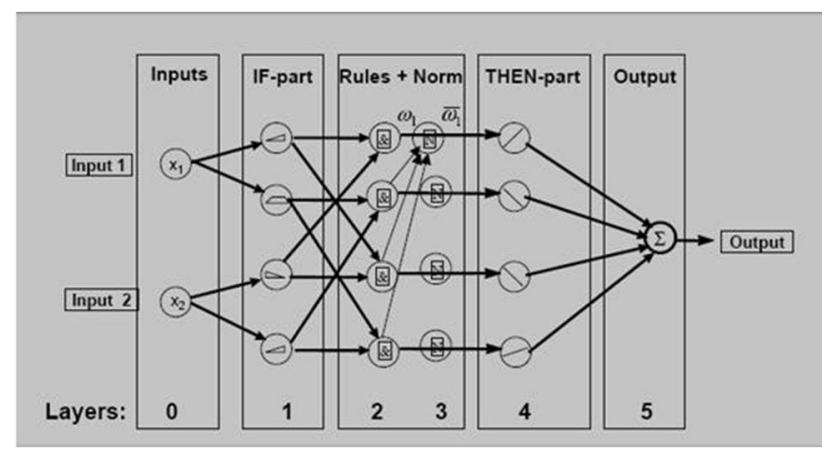

Figure 2. Structure of an ANFIS controller [14]

Two ANFIS controller were designed.

In the first case a simple solution was applied; the controller contains an ANFIS system having the error signal as input, while its output is the control signal. 9 membership functions were used for the fuzzyfication and defuzzyfication (classical ANFIS - cANFIS).

The training data contained a large recording. In this the behavior of the PID controller was taken as a sample, and the behavior of the PID controller was mimicked by the ANFIS. Measurements were taken with the PID controller at the flows of $100,300,500,750,1000$ and $1500 \mathrm{ml} / \mathrm{h}$ $[11,12]$. At each flow the input and the output of the PID controller was recorded for:

- no offset and no slope error,

- $\quad \pm 5 \mathrm{~g}$ of offset error,

- $\quad \pm 1, \pm 3, \pm 5$ and $\pm 7 \%$ of slope error,

- $\quad \pm 10 \%$ of slope error and $\pm 20 \mathrm{~g}$ of offset error.

The records were concatenated, but some structure was cut off from them. At higher errors, appeared if large control signal was necessary, the rising part of the signal was cut off (e.g. in the case of $10 \%$ of slope error and $-20 \mathrm{~g}$ of offset error the first 15-20 measurements were cut off, as this rising part was unnecessary and from the first part of the measurement when the compensation happened only those measurements were left where the control signal did not changed). Overshoots were also cut off.

As a result, the training data contained the expected behavior for high, middle and low flows and also for high, medium and low errors in various combinations. 
measurements done with the PID controller (when there was only slope error). The output was defined to reach this limit in 10-20 steps (more steps in case of high flows, less steps in case of low flows). The goal was to avoid the remained overshoot.

\section{RESULTS}

Results are presented mostly through the defined metrics from [9]. The four controllers (PID, adaptive fuzzy system, classical ANFIS, modified ANFIS) are compared through the given properties and only the one with the best performance is realized in practice in a real hemodialysis machine.

The examined properties are settling time, overshoot, accuracy and robustness measured at flows of 100,300 , 500,1000 and $1500 \mathrm{ml} / \mathrm{h}$

In order to examine the settling time $5 \mathrm{ml}$ offset error was applied simulating a static $5 \mathrm{ml}$ of volume error. $0 \%$ slope error is applied, which assumes an ideal tube segment [9]. The error was considered compensated, when the error signal first reached value 0 and did not leave its $\pm 1 \mathrm{ml}$ environment. (The $\pm 1 \mathrm{ml}$ tolerance is due to the quantitation error.) The results are shown in Table I.

TABLE I. SETTLING TIME RESULTS

\begin{tabular}{|c|c|c|c|c|c|}
\hline $\begin{array}{c}\text { Settling time } \\
{[\mathrm{s}]}\end{array}$ & $\begin{array}{c}100 \\
\mathrm{ml} / \mathrm{h}\end{array}$ & $\begin{array}{c}300 \\
\mathrm{ml} / \mathrm{h}\end{array}$ & $\begin{array}{c}500 \\
\mathrm{ml} / \mathrm{h}\end{array}$ & $\begin{array}{c}1000 \\
\mathrm{ml} / \mathrm{h}\end{array}$ & $\begin{array}{c}1500 \\
\mathrm{ml} / \mathrm{h}\end{array}$ \\
\hline PID & 888 & 296 & 184 & 92 & 65 \\
\hline AdaptiveFuzzy & 888 & 296 & 178 & 92 & 61 \\
\hline cANFIS & 888 & 296 & 177 & 96 & 68 \\
\hline mANFIS & 889 & 296 & 178 & 92 & 61 \\
\hline
\end{tabular}

Regarding the settling time, relevant difference can be found compared to $[9,10]$. This is due to the changed saturation rules, which are in the new model stricter. Furthermore, the flow dependence is closer to the real behavior of the system, which verifies the changes.

The controllers have minimal differences compared to each other. The PID controller is slower at $500 \mathrm{ml} / \mathrm{h}$ and the cANFIS is slower at $1500 \mathrm{ml} / \mathrm{h}$ than the others, but the difference is insignificant. The settling time at the lowest $(100 \mathrm{ml} / \mathrm{h})$ flow increases drastically for every controller hence, it would be advisable to decrease it.

A worst case event was set to examine the overshoot of the controllers; the tube segment was able to transfer $90 \%$ of the expected volume (-10\% slope error) and the system was also weighted with $20 \mathrm{ml}$ volume error $(20 \mathrm{ml}$ offset error). The measured overshoots are presented in Table II.

TABLE II. OVERSHOOT RESULTS

\begin{tabular}{|c|c|c|c|c|c|}
\hline $\begin{array}{c}\text { Overshoot } \\
{[\mathrm{g}]}\end{array}$ & $\begin{array}{c}100 \\
\mathrm{ml} / \mathrm{h}\end{array}$ & $\begin{array}{c}300 \\
\mathrm{ml} / \mathrm{h}\end{array}$ & $\begin{array}{c}500 \\
\mathrm{ml} / \mathrm{h}\end{array}$ & $\begin{array}{c}1000 \\
\mathrm{ml} / \mathrm{h}\end{array}$ & $\begin{array}{c}1500 \\
\mathrm{ml} / \mathrm{h}\end{array}$ \\
\hline PID & 1 & 2 & 3 & 6 & 9 \\
\hline AdaptiveFuzzy & 1 & 1 & 1 & 2 & 2 \\
\hline cANFIS & 1 & 1 & 1 & 2 & 2 \\
\hline mANFIS & 1 & 1 & 1 & 1 & 1 \\
\hline
\end{tabular}

At higher flows the PID controller had overshoot. The adaptive fuzzy system and the ANFIS controllers had only minimal overshoot, the least in case of the cANFIS

From accuracy point of view, the same settings were used as in the case of overshoot examination $(-10 \%$ slope error, $20 \mathrm{ml}$ offset error). The quantitative measurement included a $200 \mathrm{~s}$ long integration of the error signal in steady-state. Results can be seen in Table III.

TABLE III. ACCURACY MEASUREMENT RESULTS

\begin{tabular}{|c|c|c|c|c|c|}
\hline $\begin{array}{c}\text { Accuracy } \\
{[\mathrm{g} * \mathrm{~s}]}\end{array}$ & $\begin{array}{c}100 \\
\mathrm{ml} / \mathrm{h}\end{array}$ & $\begin{array}{c}300 \\
\mathrm{ml} / \mathrm{h}\end{array}$ & $\begin{array}{c}500 \\
\mathrm{ml} / \mathrm{h}\end{array}$ & $\begin{array}{c}1000 \\
\mathrm{ml} / \mathrm{h}\end{array}$ & $\begin{array}{c}1500 \\
\mathrm{ml} / \mathrm{h}\end{array}$ \\
\hline PID & -1 & -1 & 1 & 0 & 1 \\
\hline AdaptiveFuzzy & -3 & 0 & -3 & -5 & -4 \\
\hline cANFIS & -102 & -100 & -122 & -213 & -304 \\
\hline mANFIS & 0 & 2 & -1 & -2 & -2 \\
\hline
\end{tabular}

The task of the controller is not only to compensate the errors (tube segment error, volume errors, etc.), but to remove residual error in order of a strict control. The PID controller had virtually no residual error. The adaptive fuzzy system and the mANFIS had only minimal residual error that can be tolerated. The cANFIS had relevant residual error; hence, it is clear that at higher flows it was not able to keep the error at 0 levels.

Finally, from robustness point of view the controllers were checked using the same methods as in $[9,10]$. At first a sine wave was applied as weight error, which simulated periodic disturbances (e.g. shaking of the weighing scale). The amplitude was $10 \mathrm{ml}$ with the frequency of $0.25 \mathrm{~Hz}$. Secondly, an extreme disturbance out of range error was applied and a tube segment with $20 \%$ delivery error and $100 \mathrm{ml}$ of volume error. The controllers kept their stability with acceptable performance.

One relevant property of the controller is also how they can adapt to their environment. To check this property, a common tube segment was defined. In reality the tube segment has an error, meaning that this error should be compensated. The designed PID controller is not adaptive; hence, automatic adjustment is required in real time [20, 21]. It can compensate the errors, but it is unable to adapt to newer working points. On the other hand, the other three controllers (adaptive fuzzy system, cANFIS and mANFIS) were capable to adapt.

The PID controller is computationally the least expensive, the other ones consumes more power source for their operation. The adaptive fuzzy system needs empirical experiences with the pump, as these experiences are built in the fuzzy logic. The ANFIS controllers need training data for teaching and their structure has to be defined.

From the examined controllers above the best choice proved to be the mANFIS controller. It eliminates residual error; also it has no overshoot and has the same settling time as the others. Furthermore, the teaching (creating a training data set) makes it easier to modify and reuse compared to the adaptive fuzzy system. 


\section{MEASUREMENTS ON THE REAL SYSTEM}

The mANFIS controller was realized and tested on a real hemodialysis system (Fig. 4.).

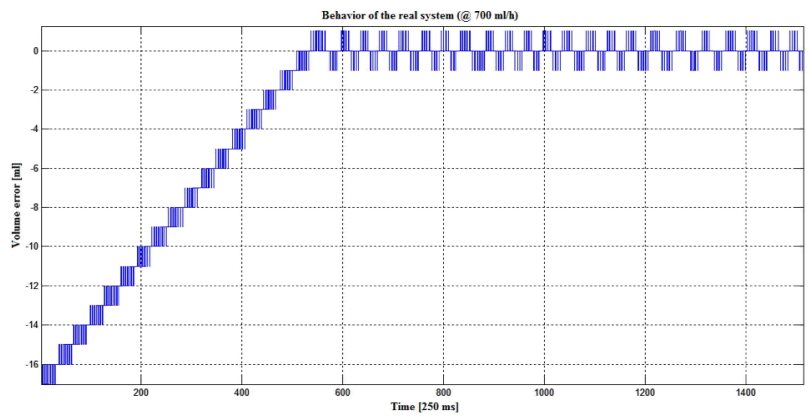

Figure 4. Behavior of the real system

The real system was weighed with $20 \mathrm{~g}$ of weight error, the tube segment had unknown delivery error. It took $127 \mathrm{~s}$ for the controller to compensate the errors and reach the steady-state. It is slightly slower than during the simulation, but this was expected similarly to $[9,10]$. In steady-state the volume error fluctuates between $\pm 1 \mathrm{ml}$ due to the high quantitation error.

For further research it would be advisable to examine the behavior of the ANFIS controller if the training data has minimal to no correlation with the PID controller. Furthermore it would be a possible upgrade the existing solution if the method described in [22] would be applied in the present controller.

\section{CONCLUSION}

In this paper soft computing control methods were compared with a classical PID controller. The PID controller and the adaptive fuzzy control system were examined in previous works as well. Some modification was applied on the simulation model, which made its behavior closer to the reality. A classical and a modified ANFIS were designed.

The properties of the controllers were very similar. They had almost the same settling time, overshoot (except PID) and accuracy (except cANFIS). However, the mANFIS proved to be the best solution as it has no overshoot, eliminates the residual error, it is adaptive and easier to train (modify and reuse) than the adaptive fuzzy system.

\section{ACKNOWLEDGMENT}

The authors are grateful for the B.Braun Medical Kft. for the support and for providing their real hemodialysis machine for measurements. The research was supported by the Hungarian OTKA projects 106392 and 105846, and project of the Vojvodina Academy of Sciences and Arts "Mathematical models of intelligent systems and theirs applications". L. Kovács is Bolyai Fellow of the Hungarian Academy of Sciences.

\section{REFERENCES}

[1] National kidney foundation, Stages of chronic kidney disease, http://www.kidney.org/atoz/content/gfr.cfm

[2] M. Misra, "The basics of hemodialysis equipment", Hemodialysis International, pp. 30-36, 2005.

[3] C. Jacobs, C. M. Kjellstrand, K. M. Koch, J. F. Winchester, "Replacement of Renal Function by Dialysis", Springer Netherlands, pp. 188-230, 333-379, 726-733, 1996.

[4] Y. M. Kadahm, "Medical Equipment II- Hemodialysis", 2012, lecture notes,

[5] N. Lamiere, R. Mehta, "Complications of Dialysis", CRC Press, pp. 29-41, 69-89, 2000.

[6] M. Gare, "Peristaltic pump: Basics and applications", http://www.buzzle.com/articles/peristaltic-pump-basics-andapplications.html , 10. 2013.

[7] SalemRepublic Rubber Company, "Peristaltic Pump Hose Tolerance Guide", http:// www.salem-republic.com

[8] M. Roberts, R. J. Winney, "Errors in fluid balance with pump control of continuous hemodialysis", The International Journal of Artificial Organs, vol. 2. pp. 99-102, 1992.

[9] J. Klespitz, L. Kovács. "Identification and control of peristaltic pumps in hemodialysis machines." Computational Intelligence and Informatics (CINTI), 2013 IEEE 14th International Symposium on. IEEE, 2013.

[10] J. Klespitz, M. Takács, L Kovács. "Application of fuzzy logic in hemodialysis equipment." Intelligent Engineering Systems (INES), 2014 IEEE 18th International Symposium on. IEEE, 2014.

[11] C. Ronco, et al. "Effects of different doses in continuous venovenous haemofiltration on outcomes of acute renal failure: a prospective randomised trial." The Lancet 356.9223 (2000): 2630.

[12] National Kidney Foundation Kidney Disease Outcomes Qualitiy Initiative (NKF KDOQI),

http://www.kidney.org/professionals/kdoqi/guidelines_commentar ies.cfm

[13] J-SR. Jang, "ANFIS: adaptive-network-based fuzzy inference system "Systems, Man and Cybernetics, IEEE Transactions on 23.3 (1993): 665-685.

[14] Copyright by Piero P. Bonissone, 2002, Creative Commons Attribution 3.0 Licence, http://en.wikipedia.org/wiki/File:Anfis.JPG

[15] K. Kabini, "Review of ANFIS and its Application in Control of Machining Processes." Sustainable Research and Innovation Proceedings 3 (2011).

[16] B. Lantos, "Fuzzy systems and genetic algorithms." Müegyetemi Kiadó, Budapest, Hungary (2002).

[17] HS. Ahn, YQ. Chen, K. L. Moore. "Iterative learning control: brief survey and categorization." IEEE Transactions on Systems Man and Cybernetics part C Applications and Reviews 37.6 (2007): 1099.

[18] Y. Wang, F. Gao, F. J. Doyle III. "Survey on iterative learning control, repetitive control, and run-to-run control." Journal of Process Control 19.10 (2009): 1589-1600.

[19] JX. Xu, Y Tan. "Linear and nonlinear iterative learning control." Vol. 291. Berlin: Springer, 2003.

[20] K. J. Åström, B. Wittenmark. Adaptive control. Courier Dover Publications, 2013.

[21] I. D. Landau et Al., "Adaptive Control", Communications and Control Engineering, Springer - Verlag, London, 2011.

[22] YC. Wang, CJ. Chien. "Fuzzy-neural adaptive iterative learning control for a class of nonlinear discrete-time systems." Fuzzy Theory and its Applications (iFUZZY), 2012 International Conference on. IEEE, 2012. 